\title{
BMJ Open Parenting roles and knowledge in neonatal intensive care units: protocol of a mixed methods study
}

\author{
Elisabete Alves, ${ }^{1,2}$ Mariana Amorim, ${ }^{1}$ Sílvia Fraga, ${ }^{1,2}$ Henrique Barros, ${ }^{1,2}$ \\ Susana Silva ${ }^{1,2}$
}

To cite: Alves $\mathrm{E}$, Amorim M, Fraga $\mathrm{S}$, et al. Parenting roles and knowledge in neonatal intensive care units: protocol of a mixed methods study. BMJ Open 2014:4:e005941. doi:10.1136/bmjopen-2014005941

- Prepublication history and additional material for this paper is available online. To view these files please visit the journal online (http://dx.doi.org/10.1136/ bmjopen-2014-005941).

Received 19 June 2014 Accepted 25 June 2014

\section{CrossMark}

For numbered affiliations see end of article.

Correspondence to Professor Susana Silva; susilva@ispup.up.pt

\section{ABSTRACT}

Introduction: There is a strong focus on the translation of scientific knowledge into evidence-based practice when dealing with very preterm births. The aim is to standardise and rationalise healthcare. The incorporation of parents' perspectives with respect to the organisation of care and technical interventions in neonatal intensive care units (NICUs) is needed. This study aims to analyse the repertoire of meanings, knowledge and emotions actualised by the parents of very preterm infants hospitalised in NICUs in the decision process regarding parental care, treatment options and uses of information sources.

Methods and analysis: This is a mixed-methods, observational study. The methodological strategy will rely on: (1) Ethnographic observation, carried out in a level III NICU located in the North of Portugal, during 6 months; (2) NICU-based surveys of mothers and fathers of very preterm infants born between July 2013 and June 2014 and admitted at the seven public level III NICUs of the Northern Health Region of Portugal; (3) Single and couple semistructured interviews to a subsample of mothers and fathers of very preterm infants, 4 months after birth. Inferential statistics will be used to analyse the quantitative data and content analysis, with an iterative and reflexive process and will be implemented to assess qualitative data.

Ethics and dissemination: The study protocol was approved by the National Data Protection Commission and the Ethics Committee of all the hospitals involved. The current project will contribute to develop resources for enriched good medical practices in the context of neonatal services through integrating insights from social sciences, public health, epidemiology and ethics. The expected dissemination actions are effective tools in designing strategies that aim to develop familycentred care and to improve medical practices in the context of neonatal services.

\section{INTRODUCTION}

Very preterm deliveries are those that occur between 22 and 31 weeks of gestation. ${ }^{1}$ These children are very fragile and dependent on intensive care. The percentage of live births with a gestational age $<32$ weeks varied

\section{Strengths and limitations of this study}

- The study will serve as the foundation for a set of integrated insights related to the perspectives of parents of children born very prematurely on strategies to manage their roles and knowledge in a highly technological sociomedical environment.

- The study protocol was approved by the National Data Protection Commission and the Ethics Committee of all the hospitals involved.

- The expected dissemination actions can be useful and effective tools in designing strategies that aim to develop family-centred care and to improve medical practices in the context of neonatal services.

from $0.7 \%$ to $1.4 \%$ in Europe in $2010 .^{2} 3$ Lower proportions of very preterm live birth (below 1.0\%) were observed in Iceland, France, Malta, Finland, Lithunia, Sweden and the region of Wallonia/Belgium; higher rates (above 1.2\%) were registered in Hungary and the region of Brussels/Belgium (1.4\%), Germany and Austria (1.3\%). ${ }^{2}$ In Portugal, $1.0 \%$ of live births in 2010 were very preterm. ${ }^{2}$

Prematurity results from multiple causes such as maternal biogenetic, pregnancy history, sociodemographic and psychological characteristics (eg, pre-eclampsia or eclampsia, intrauterine growth restrictions, uterine overdistension, uterine contractions, short cervical length, periodontal disease, vascular disease, low maternal body-mass index, previous preterm birth, artificially conceived pregnancy, close temporal proximity to a previous delivery, low socioeconomic and educational status, extreme maternal ages, single marital status, type of work and level of physical activity, and absence of a strong supportive economic and social safety net). ${ }^{5}$

Outcomes of very preterm infants concerning survival rates, mortality and neurological and respiratory morbidity differ between neonatal intensive care units (NICUs) and between 
European regions, according to varied uses of medical interventions and several approaches to the organisation of care. $^{67}$ The MOSAIC study showed crude in-hospital mortality rates ranging from $7.9 \%$ to $24.7 \%$ between 10 European regions, presenting an average rate of $14.2 \%$. The North of Portugal had a rate of in-hospital mortality of very preterm infants of $15.9 \%$, lower than the regions of Flanders/Belgium, Lazio/Italy and Wielkopolska and Lubuskie/Poland, but higher than Hesse/Germany, Northern UK and Eastern Denmark. ${ }^{8}$ Despite significant medical and technological advances in recent decades, infants born before 32 weeks of gestation remain at high risk of death and neurodevelopmental impairment, including cerebral palsy, mental retardation and sensory loss. ${ }^{6}$

Time is ripe to develop optimal evidence-based effective perinatal intensive care ${ }^{9}$ by standardising approaches to the organisation of care and medical interventions, especially in the case of prematurity. ${ }^{6}$ There is a strong focus on the translation of scientific knowledge into evidence-based practice when dealing with very preterm births. The incorporation of parents' perspectives with respect to the organisation of care and technical interventions in NICU is needed. ${ }^{10} 11$

Evidence about family-centred care, ${ }^{12}$ defined as provision of care that is respectful of and responsive to individual parents' preferences, needs and values is essential. ${ }^{13}{ }^{14}$ However, the literature on coconstitution of parenting (mothering and fathering) and medical technologies, as well as the biomedical and embodied knowledge in the context of prematurity is scarce, focusing on the following main domains: informed consent and perceptions of risk; ${ }^{15}$ needs of information and communication; ${ }^{16}$ parental stress; ${ }^{17}$ psychopathological symptoms; ${ }^{18} 19$ meanings of acceptable quality of future life (of offspring) and legitimacy of end-of-life decisions; ${ }^{20}$ and breastfeeding. ${ }^{21}$

This research project is concerned with the societal, ethical and practical impacts of medical technology in society, by focusing the specific case of parents' experiences in NICUs. Building on the interdisciplinary competencies of the project team, the study will serve as a foundation for a set of integrated insights related to the perspectives of parents of children born very prematurely on strategies to manage their roles and knowledge in a highly technological sociomedical environment. The present study aims to analyse the repertoire of meanings, knowledge and emotions actualised by the parents of very preterm infants hospitalised in NICUs in the decision process regarding parental care, treatment options and uses of information sources, in order to enrich good medical practices, improve health governance and develop family-centred care.

The specific objectives of this project are organised around the identification and understanding of:

1. Parents' proposals to achieve changes in the delivery of healthcare in neonatal services with respect to the organisation of care and technical interventions.

2. Broad patterns of parents' social, demographic, psychological and medical characteristics within the NICU.
3. Expectations, responsibilities and knowledge actualised by parents to understand the actual and future health status of their very preterm infants.

4. Representations and practices concerning parental roles, rights and duties enacted by mothers and fathers of very preterm children in the context of the NICU.

5. To evaluate the impacts of being a mother or a father of an 'unhealthy' baby in daily lives, identities and social position in the community.

\section{METHODS AND ANALYSIS}

This is a mixed-methods, observational study. The methodological strategy will rely on: (1) Ethnographic observation in a level III NICU located in the North of Portugal for a duration of 6 months; (2) NICU-based surveys of parents of very preterm infants admitted at the seven public level III NICUs of the Northern Health Region of Portugal over a 12 month period; and (3) Single and couple semistructured interviews to a subsample of mothers and fathers of very preterm infants 4 months after birth.

\section{Ethnographic research}

Fieldwork will be carried out in the level III NICU with the largest number of very preterm infants located in the North of Portugal ${ }^{22}$ for a duration of 6 months. In this setting, interactions between (1) mothers and fathers of very preterm children, (2) parents and infants and (3) parents and staff (neonatologists and nurses) will be observed. The physical spaces and the organisation of care will also be analysed. The researcher will attend meetings such as at nurses' shifts, parents' reunions and conversations between staff members and parents and between mothers and fathers, but will not participate in the medical procedures and the decisionmaking. During the fieldwork, the researcher will use a white coat, according to the NICU's mandatory rules.

Data will be collected over a period of approximately 100 contact hours, at different periods of the day (morning, afternoon and night) and on weekdays and weekends. All mothers, fathers and health professionals will be considered potential informants. Descriptions and reflexive analysis of all the observations will be taped and transcribed.

Taking into account the specific objectives of the project, the literature review and the first field diaries, an observation grid will be constructed with at least five dimensions: interpersonal relationships (mother-father, mother/father-infant, mother/father-staff; mother/ father-peers; staff-staff and staff-infant), healthcare organisation (medical care, hygienic care, feeding, cuddling and teaching the parents), sources of information (available in the NICU and used by the parents), spaces (outside and inside the NICU) and personal reflections. 


\section{NICU-based surveys}

The estimated number of very preterm children in the North of Portugal is about 250 per year, ${ }^{22}$ and all these babies must be admitted in public NICUs. All mothers and fathers of very preterm infants born between 1 July 2013 and 30 June 2014 and hospitalised at the seven public level III NICUs of the Northern Health Region of Portugal (Centro Hospitalar de S João, Centro Hospitalar de Vila Nova de Gaia/Espinho, Centro Hospitalar do Alto Ave, Centro Hospitalar do PortoUnidade Maternidade de Júlio Dinis, Hospital de Braga, Centro Hospitalar entre Douro e Vouga and Unidade Local de Saúde de Matosinhos) will be invited to integrate the study. Parents will be approached during the hospital stay by a health professional of the NICU (neonatologist or nurse), who will be responsible for the study presentation and invitation. We expect a participation rate higher than $90 \%$.

Only parents with infants born before 32 weeks of gestation and still hospitalised in the aforementioned NICUs at the time of the interview will be considered eligible to integrate the study. Parents with serious illness that precluded NICU visitation (eg, severe chronic conditions), families who are absent in NICU during the hospitalisation period and parents whose infants are discharged or transferred to another hospital will be excluded.

The recruitment of the study participants will take place 15-22 days after birth, during hospitalisation in the NICU. Trained interviewers will be responsible for conducting face-to-face interviews, using structured questionnaires, with mothers and fathers together and separately, although in approximate times.

Data on demographic and socioeconomic characteristics (age, country of origin, marital status, years of formal education, occupation, income, subjective social class and religious beliefs), personal medical history (diagnosis of depression, hypertension, dyslipidaemia, diabetes mellitus, myocardial infarction, stroke, heart failure and cancer), gynaecological and obstetric history (number of previous pregnancies, obstetrics results, time taken to get pregnant, use of assisted reproductive technologies, number of offspring and previous hospitalisation in an NICU), lifestyles (smoking and alcohol intake), anthropometrics (self-reported weight before and at the end of pregnancy and height), breast milk supply, perception of factors that help or hinder breastfeeding in the NICU and main sources of information about the infant's hospitalisation will be collected. Participants will be asked to report prepregnancy smoking and alcohol intake, as well as changes implemented during pregnancy (cessation, reduction or increase) and current consumption after birth.

Thereafter, mothers and fathers will be asked to fill a form on quality, utility and accessibility of specific sources of information, the Multidimensional Scale of Perceived Social Support, ${ }^{23}$ the NICU Family Needs Inventory ${ }^{24}$ and the Parental Stress Scale in the NICU. ${ }^{25}$
Clinical records of NICU hospitalisation will be reviewed by the interviewers to retrieve data on pregnancy complications (gestational hypertensive disorders, gestational diabetes and placental abnormalities), type of delivery and neonatal characteristics (birth weight and height, gestational age and cephalic perimeter) of very preterm infants.

\section{Qualitative interviews}

Approximately 4 months after birth, a subsample of mothers and fathers will be contacted to participate in single and couple semistructured interviews. The interviews will enable researchers to pursue a deeper insight into the complexity of, and similarities and differences between, the views and experiences of the mothers and fathers of very preterm infants around their roles and knowledge.

We will recruit approximately eight mothers, eight fathers and 30 couples, purposively sampled to include parents of babies with birth weight (1) $<1000 \mathrm{~g}$ (extremely low birth weight) and $(2) \geq 1000 \mathrm{~g} .{ }^{26}$ In addition, a heterogeneity sampling will be considered for maximum variation of views and experiences, until reaching thematic saturation.

Interviews will take place at NICUs, in participant homes, or at other locations of the participant's choosing and, following consent, will be digitally recorded. The topic guide will include the following areas: how parents of very preterm children deal with uncertainty and doubts and how they made their decisions concerning parental care (namely in the fields of physical contact and breastfeeding), treatment options and uses of information sources; their views of the consent processes; their understandings of medical facts, of technologies applied to perinatal care and of prognosis; their views of life and living with handicaps; information and communication needs of parents; and their wider awareness of social and ethical issues in this area.

All interviews will be transcribed verbatim. Interviewing and preliminary analysis will be carried out by one of the trained interviewers who apply the questionnaire at birth, so that consistent training and quality assurance are guaranteed.

\section{Data analysis}

\section{Quantitative data}

All statistical analyses will be performed using Stata V.11.0 (College Station, Texas, USA, 2009) and inferential statistics will be used to answer the objectives of this project.

Data will be described as counts and proportions for categorical variables, mean and SD for normally distributed continuous variables and median and IQR for nonnormally distributed continuous variables. The prevalence of the outcomes will be presented with $95 \%$ CI.

According to the specific objectives established, different analytic approaches will be considered. Means will be compared with the Student t or Mann-Whitney tests 
as appropriate. For categorical variables, differences in frequencies and proportions will be assessed using $\chi^{2}$ test or Fisher's exact test.

The associations between explanatory variables and the outcomes will be estimated by crude and adjusted prevalence ratios (PR) and respective 95\% CI using robust Poisson regression or by OR and respective $95 \%$ CI using logistic regression. The PR is more conservative, consistent and interpretable than the OR when estimates of prevalence are to be compared between groups ${ }^{27} 28$ and, for this reason will be privileged, whenever adequate.

\section{Qualitative data}

We will use traditional content analysis with an iterative and reflexive process. The findings will be inductively derived directly from the analysis of the raw data.

Field notes from ethnographic research as well as single and couple semistructured interviews will be analysed using NVivo10 (QSR International, USA, 2013). Through the hermeneutic analysis of transcripts, using constant comparison and category building procedures, major themes will be identified. We will code data as we collect it to inform the direction of subsequent interviews, coding and case selection.

Based on the protocol for content analysis developed by Stemler ${ }^{29}$ an independent analysis will be conducted by two researchers to identify, quotation by quotation, all the perspectives of parents of very preterm infants regarding their experiences, knowledge and decisionmaking actualised during hospitalisation in the NICU. These quotations will be then synthesised into categories, defined as 'a group of words with similar meaning and connotations', and the number of interviews where each category emerged will be recorded. Then, such categories will be grouped in themes, according to the protocol for thematic analysis developed by Mays et al. ${ }^{30}$ Disagreements in abstractions will be discussed with a third researcher and resolved by consensus. We will seek respondent validation on emerging conclusions, and maximise internal reliability and reflection though comparing coding between multiple researchers.

\section{Ethics and dissemination}

The study protocol was approved by the National Data Protection Commission and the Ethics Committee of all the seven hospitals where the study will be conducted.

Procedures will be developed in order to guarantee data confidentiality and protection. Each NICU and each participant will be identified with a numerical code. All participants will receive an information sheet with an explanation on the purposes and design of the study. Each participant will provide prior consent to obtain personal contacts, collect information from clinical records and interview recordings. All materials will be anonymous and confidential. Data protection will be guaranteed in accordance with the usual rules of confidentiality and only the research team will have access to the data. Personal data, consents, questionnaires, interview tapes and transcripts will be coded and kept separately from one another in locked file cabinets. All audio files will be destroyed at the end of the study. Interview transcripts will be archived by ISPUP for 5 years. Once archived, transcripts will be subject to strict protection and will not be made available, unedited, to any second party.

All interviewers will be trained using a structured protocol addressing all the questionnaires' queries and periodic supervision of their work will be undertaken. A multidisciplinary team, with experience in national and international projects, will be responsible for the staff training and the development of the questionnaire and the interview guide. Transcription of the interviews will be performed by a professional, reliable service with a strict confidentiality policy in operation. Identifiable information will inevitably be captured on the audio recordings but only the research team and transcription service will have access to these files.

Drawing on robust societal approaches to parents' knowledge and experiences, this study intends to contribute for more effective and transparent forms of intensive neonatal care. This project is groundbreaking for several reasons; of particular interest is the inclusion of fathers in the research and the interviewing of both parents in two different settings-single and couple, and during their babies' time in the NICU instead of only later in retrospect; the provision of evidence on intervention strategies to manage knowledge and information and communication needs and to develop theoretical accounts grounded in research data; and the exploration of the influence of the cultural background and the social networks of the parents in their experiences, knowledge, roles and views around participation in decision-making, organisational rules and regulations within the NICU.

The current project will contribute to enrich good medical practices in the context of neonatal services through integrating insights from social sciences, public health, epidemiology and ethics. It is expected to generate and disseminate evidence on parents of very preterm babies' roles, experiences, views and knowledge regarding neonatal care, information and communication needs, perception of risks, informed consent practices and stigmatisation, providing information directed to clinical scientists, practitioners, policymakers and social scientists. The expected dissemination actions can be useful and effective tools in designing strategies that aim to develop family-centred care and to improve medical practices in the context of neonatal services.

Results from this project will be published in international and national peer-reviewed journals, separately and in concluding papers combining data across methodologies. Moreover, key results will be presented at international conferences, as well as at national seminars aimed at practitioners, policymakers and postgraduate students in order to debate implications for familycentred policies and care, and ethics in clinical practice. The study will also contribute to the advance training of 
young researchers through a postdoctorate fellowship and the production of master theses. Finally, dissemination of results among mothers and fathers of very preterm infants will be accomplished through the organisation of one science shop.

\section{Author affiliations}

${ }^{1}$ Institute of Public Health-University of Porto (ISPUP), Porto, Portugal

${ }^{2}$ Department of Clinical Epidemiology, Predictive Medicine and Public Health, University of Porto Medical School, Porto, Portugal

Contributors SS and HB conceived the research questionnaire. EA, MA, SF and SS drafted the quantitative and qualitative aspects of the study protocol. EA was responsible for drafting this paper and all authors read, provided important revisions and approved the final version of the manuscript.

Funding This study is supported by FEDER funding from the Operational Programme Factors of Competitiveness - COMPETE and by national funding from the FCT-Foundation for Science and Technology (Portuguese Ministry of Education and Science) within the project 'Parenting roles and knowledge in neonatal intensive care units' (ref. PTDC/CS-ECS/120750/2010-FCOMP-010124-FEDER-019902), and the Starting Grant IF/00956/2013 (to SS).

Competing interests None.

Patient consent Obtained.

Ethics approval The Study protocol was approved by the National Data Protection Commission and the Ethics Committee of all the seven hospitals where the study will be conducted.

Provenance and peer review Not commissioned; peer reviewed for ethical and funding approval prior to submission.

Open Access This is an Open Access article distributed in accordance with the Creative Commons Attribution Non Commercial (CC BY-NC 4.0) license, which permits others to distribute, remix, adapt, build upon this work noncommercially, and license their derivative works on different terms, provided the original work is properly cited and the use is non-commercial. See: http:// creativecommons.org/licenses/by-nc/4.0/

\section{REFERENCES}

1. World Health Organization (WHO). Born too soon: the global action report on preterm birth. Geneva: WHO Press, 2012.

2. Field D, Draper ES, Fenton A, et al. Rates of very preterm birth in Europe and neonatal mortality rates. Arch Dis Child Fetal Neonatal Ed 2009:94:F253-6.

3. Zeitlin J, Mohangoo A, Delnord M. The European Perinatal Health Report: health and care of pregnant women and babies in Europe in 2010 [22nd Feb 2014]. http://www.europeristat.com/reports/ european-perinatal-health-report-2010.html.

4. Goldenberg RL, Culhane JF, lams JD, et al. Epidemiology and causes of preterm birth. Lancet 2008;371:75-84.

5. Berkowitz GS, Papiernik E. Epidemiology of preterm birth. Epidemio Rev 1993:15:414-43.

6. Milligan DW. Outcomes of children born very preterm in Europe. Arch Dis Child Fetal Neonatal Ed 2010:95:F234-40.

7. Draper ES, Zeitlin J, Fenton AC, et al. Investigating the variations in survival rates for very preterm infants in 10 European regions: the MOSAIC birth cohort. Arch Dis Child Fetal Neonatal Ed 2009;94: F158-63.

8. Zeitlin J, Draper ES, Kollee L, et al. Differences in rates and short-term outcome of live births before 32 weeks of gestation in
Europe in 2003: results from the MOSAIC cohort. Pediatrics 2008;121:e936-44.

9. EPICE. Effective perinatal intensive care in Europe: translating knowledge into evidence-based practice 2014 [22nd Feb 2014] http://www.epiceproject.eu/pt

10. Jacono J, Hicks $\mathrm{G}$, Antonioni $\mathrm{C}$, et al. Comparison of perceived needs of family members between registered nurses and family members of critically ill patients in intensive care and neonatal intensive care units. Heart Lung 1990;19:72-8.

11. Timmermans S, Berg M. The gold standard: the challenge of evidence-based medicine and standardization in health care. Philadelphia, PA: Temple University Press, 2003.

12. Malusky SK. A concept analysis of family-centered care in the NICU. Neonatal Netw 2005;24:25-32.

13. European Commission. Eurobarometer Qualitative Study. Patient involvement. Aggregate report; 2012. http://ec.europa.eu/health/ healthcare/docs/eurobaro patient involvement 2012 en.pdf

14. WHO. People-centred health care: a policy framework. Geneva: World Health Organization, 2007.

15. Alderson P, Hawthorne J, Killen M. Parents' experiences of sharing neonatal information and decisions: consent, cost and risk. Soc Sci Med 2006;62:1319-29.

16. De Rouck S, Leys M. Information needs of parents of children admitted to a neonatal intensive care unit: a review of the literature (1990-2008). Patient Educ Couns 2009;76:159-73.

17. Howe TH, Sheu CF, Wang TN, et al. Parenting stress in families with very low birth weight preterm infants in early infancy. Res Dev Disabil 2014;35:1748-56.

18. Garel M, Bahuaud M, Blondel B. [Consequences for the family of a very preterm birth two months after discharge. Results of the EPIPAGE qualitative study]. Arch Pediatr 2004;11: 1299-307.

19. Vigod SN, Villegas L, Dennis CL, et al. Prevalence and risk factors for postpartum depression among women with preterm and low-birth-weight infants: a systematic review. BJOG 2010;117:540-50.

20. Vermeulen E. Dealing with doubt: making decisions in a neonatal ward in The Netherlands. Soc Sci Med 2004;59:2071-85.

21. Alves $\mathrm{E}$, Rodrigues $\mathrm{C}$, Fraga $\mathrm{S}$, et al. Parents' views on factors that help or hinder breast milk supply in neonatal care units: systematic review. Arch Dis Child Fetal Neonatal Ed 2013;98:F511-17.

22. EPICE Group Portugal. Newsletter 62014 [15th May 2014]. http:// issuu.com/ispup/docs/newsletter_epice-po_n.6

23. Zimet GD, Powell SS, Farley GK, et al. Psychometric characteristics of the multidimensional scale of perceived social support. $J$ Pers Assess 1990;55:610-17.

24. Ward K. Perceived needs of parents of critically ill infants in a neonatal intensive care unit (NICU). Pediatr Nurs 2001; 27:281-6.

25. Souza SRd, Dupas G, Balieiro MMFG. Adaptação cultural e validação para a língua portuguesa da Parental Stress Scale: Neonatal Intensive Care Unit (PSS:NICU). Acta Paulista de Enfermagem 2012;25:171-6.

26. WHO. International statistical classification of diseases and related health problems-10th revision. 2011 [22nd Feb 2014]. http://www. who.int/classifications/icd//CD10Volume2_en_2010.pdf.

27. Thompson ML, Myers JE, Kriebel D. Prevalence odds ratio or prevalence ratio in the analysis of cross sectional data: what is to be done? Occup Environ Med 1998;55:272-7.

28. Barros AJ, Hirakata VN. Alternatives for logistic regression in cross-sectional studies: an empirical comparison of models that directly estimate the prevalence ratio. BMC Med Res Methodol 2003:3:21.

29. Stemler S. An overview of content analysis. Pract Asses Res Eval 2001;7:1-9.

30. Mays N, Pope C, Popay J. Systematically reviewing qualitative and quantitative evidence to inform management and policy-making in the health field. J Health Serv Res Policy 2005;10(Suppl 1):6-20. 